\title{
SEIS SIGMA: CARACTERÍSTICAS DO PROGRAMA EM ONZE EMPRESAS SITUADAS NA REGIÃO METROPOLITANA DE PORTO ALEGRE
}

\author{
SIX SIGMA: PROGRAM CHARACTERISTICS IN ELEVEN \\ COMPANIES LOCATED IN THE METROPOLITAN REGION OF \\ PORTO ALEGRE
}

\author{
Jaqueline T. M. Corrêa RodriguesE-mail: jaque@producao.ufrgs.br \\ Liane Werner*E-mail: liane@producao.ufrgs.br \\ * Universidade Federal do Rio Grande do Sul, UFRGS, Porto Alegre, RS
}

\begin{abstract}
Resumo: O programa Seis Sigma alia fatores gerenciais com ferramentas estatísticas e está implantado em várias empresas atualmente. O Rio Grande do Sul é um estado brasileiro que apresenta grande industrialização, principalmente na região metropolitana de Porto Alegre, e algumas destas indústrias já implantaram o programa Seis Sigma. O objetivo deste artigo é apresentar algumas características gerais do programa Seis Sigma em empresas localizadas nesta região, através de um estudo exploratório. A pesquisa foi iniciada com uma revisão bibliográfica sobre o programa, que subsidiou a elaboração do questionário. Onze empresas participaram da pesquisa em julho e agosto de 2008. Foram utilizadas ferramentas estatísticas e análises qualitativas para análise dos resultados. A maioria das empresas que participou da pesquisa é classificada como de grande porte e utiliza o método DMAIC para implementar o Seis Sigma. A busca pela melhoria dos processos foi apontada como causa de implantação do programa em grande parte das empresas e em somente uma empresa participante o Seis Sigma está implantado em todos os setores, incluindo áreas administrativas. Outro resultado a ser salientado é a falta de critérios claros para definição do quadro de especialistas e a falta de uniformização da formação acadêmica para estes especialistas.
\end{abstract}

Palavras-chave: Seis Sigma. Região Metropolitana de Porto Alegre.

Abstract: Six Sigma combines managerial factors with statistical tools and is deployed in many enterprises today. Rio Grande do Sul is a Brazilian state that has a great industrialization, especially in the metropolitan area of Porto Alegre. Some of these industries have deployed the Six Sigma program. The aim of this paper is to present characteristics of the Six Sigma program in companies located in this region, through an exploratory study. A literature review subsidized the development of the questionnaire. Eleven companies participated of research in July and August 2008. It used statistical tools and qualitative analysis to analyze the results. Most of the companies that participated is classified as large and uses the DMAIC method to implement Six Sigma. The principal cause of program implementation was process improvement. In only one company the Six Sigma is implemented in all sectors, including administrative areas. Another result is the lack of clearly criteria for defining the career of Belts and the discrepancy of academic formation of these specialists.

Keywords: Six Sigma. Metropolitan region of Porto Alegre.

\section{INTRODUÇÃO}

Mesmo antes da Revolução Industrial, os artesãos e mestres reconheciam a 
importância da qualidade de seus produtos. No século $X X$, a busca pela qualidade passou a ser essencial para a sobrevivência das empresas num mundo cada vez mais competitivo. Nos anos 20 implementou-se o Controle de Qualidade utilizandose os gráficos de controle de Shewart para analisar a variabilidade estatística de variáveis críticas dos produtos. (KESSLER, 2004).

Muitas foram as contribuições ao longo destes anos para se atingir o conceito atual de Qualidade. Feigenbaun introduziu o conceito de Controle de Qualidade Total (TQM - Total Quality Management). Edward Deming reforça a idéia de que a qualidade e a produtividade aumentam à medida que se reduz a variabilidade dos processos produtivos. Joseph Juran definiu um produto ou serviço de qualidade como aquele que atende de forma satisfatória os clientes. Kaoru Ishikawa sugeriu que a participação dos trabalhadores é essencial para a implementação bem sucedida de TQM, desenvolvendo o conceito dos círculos de controle de qualidade. Philip Crosby deu atenção aos custos da qualidade em seu trabalho e propondo a adoção do padrão de desempenho baseado no zero defeito (SLACK et al., 2006; JURAN, 1995).

Assim, alguns modelos de gestão de qualidade surgiram nos últimos tempos, como a série ISO 9000. Um programa que está em alta evidência é o Seis Sigma, baseando-se em características do TQM e no alinhamento de projetos com as estratégias da empresa, busca elevar a relação custo-benefício dos projetos (PINTO; CARVALHO; HO, 2006).

O Rio Grande do Sul (RS) é amplamente industrializado e foi classificado em $4^{\circ}$ lugar entre os Estados brasileiros em relação ao PIB - Produto Interno Bruto - em 2006 (FEE, 2007). O Estado também apresentou crescimento da sua atividade industrial (IDI - Índice de Desenvolvimento Industrial) pelo terceiro mês seguido no mês de Novembro de 2009, segundo a FIERGS (2009), mostrando capacidade de recuperação da atividade produtiva após a crise mundial que afetou o mundo em 2008 e 2009

Considerando que o Seis Sigma é um programa relativamente recente, ainda não se realizaram muitas pesquisas no Brasil sobre o tema. A maioria das pesquisas realizadas objetiva traçar o perfil do programa nas empresas que estão localizadas na região Sudeste do país, como as realizadas por Andrietta e Miguel (2007), Carvalho, Ho e Pinto (2007), Mergulhão (2003) e Oliveira e Daher (2004). Desta forma, o objetivo deste artigo é mapear características gerais do programa Seis 
Sigma em onze empresas situadas na região metropolitana de Porto Alegre a fim de ampliar os estudos sobre o tema no Brasil e comparar estes resultados com os similares apresentados por outros pesquisadores.

\section{O SEIS SIGMA}

O Seis Sigma pode ser entendido sob vários pontos de vista. Para alguns autores o programa tem caráter gerencial, enquanto para outros o conteúdo estatístico é que ganha destaque (WERKEMA, 2004; ECKES, 2003; ANTONY e BANUELAS, 2002; PEREZ-WILSON, 1999). Há ainda os autores que o consideram o Seis Sigma como resultado de uma combinação entre os fatores gerenciais e estatísticos, como Antony (2004), que define o programa como uma estratégia poderosa que emprega uma abordagem disciplinada para lidar com a variabilidade dos processos e utiliza ferramentas estatísticas e técnicas de gestão bem estruturadas e baseadas em dados quantitativos.

Como o Seis Sigma utiliza ferramentas estatísticas conhecidas, o programa parece não envolver nada de novo, porém Werkema (2004) salienta que a abordagem e a forma de implementação do programa são únicas. O programa Seis Sigma tem como diferencial em relação aos demais programas de qualidade, conforme Antony (2004), o foco nos resultados financeiros, a importância dada à liderança para alcance do sucesso do programa, além da integração entre elementos humanos (cultura organizacional, clientes, funcionários...) e elementos de processo (ferramentas, dados, medidas...) para solução de problemas.

Santos e Martins (2008) afirmam que o Seis Sigma está se consolidando como uma forma de promover a melhoria do desempenho do negócio da empresa, aumentar a competitividade e impulsionar ações estratégicas e gerenciais que priorizem a melhoria contínua de produtos e serviços, incrementar a capacidade de inovação e reduzir custos. Para Eckes (2003) o Seis Sigma deve estar diretamente relacionado com a estratégia da empresa, e, por este motivo, o programa é de responsabilidade da alta administração da empresa, uma vez que ela é que define as estratégias e a forma como o Seis Sigma será implementado na empresa.

O programa Seis Sigma é composto de vários projetos. Estes projetos são criados para solucionar problemas ou gerar melhorias nos processos. Para Cabrera Jr. (2006), o sucesso dos projetos é sustentado por dois pilares: o conhecimento 
técnico (ferramentas) e nos recursos humanos (pessoas).

Uma organização para ser denominada Seis Sigma deve inserir as práticas e conceitos do Seis Sigma em suas atividades gerenciais diárias e apresentar melhorias significativas no desempenho do processo e na satisfação dos clientes (PANDE et al., 2001). Os autores salientam que simplesmente usar medidas sigma e ferramentas estatísticas não qualifica a organização como sendo Seis Sigma e esclarecem que não é preciso alcançar os níveis reais de desempenho Seis Sigma em nenhum projeto, mas deve-se ter isto como objetivo.

Para concretizar o programa Seis Sigma nas organizações, segundo PerezWilson (1999), é preciso adotar uma metodologia consistente, clara, concisa e compreensível. Werkema (2004) e Eckes (2003) não utilizam o termo metodologia, e sim método estruturado e tático, respectivamente. O método mais difundido, conforme a literatura citada neste trabalho é o DMAIC, que, segundo Werkema (2004), é constituído de cinco etapas: Define (Definir); Measure (Medir); Analyze (Analisar); Improve (Melhorar); e Control (Controlar).

A primeira etapa do DMAIC é o Define, que consiste em descrever o problema, selecionar qual projeto será utilizado para solucionar este problema e definir a meta que deverá ser alcançada (WERKEMA, 2004; ECKES, 2003). Para Werkema (2004), descrever o problema é avaliar seu histórico, seu impacto sobre os clientes, sobre a estratégia e sobre os resultados financeiros da empresa. Algumas ferramentas como Project Charter, cartas de controle e análises econômicas são utilizadas nesta fase.

Measure é a segunda etapa do DMAIC. Nesta fase devem ser definidas quais as características do projeto que deverão ser monitoradas, de que forma os dados serão obtidos e registrados e quais as especificações do projeto (ECKES, 2003). Ferramentas estatísticas como estratificação, folha de verificação, diagrama de Pareto, histograma e cartas de controle são úteis para que se possa analisar o problema em estudo (WERKEMA, 2004).

Com os dados obtidos na segunda etapa, o passo seguinte é a etapa Analyze. Segundo Eckes (2003), durante esta etapa são analisados os dados e os processos envolvidos e determinadas quais as causas que contribuem para o baixo desempenho do processo. Para analisar os dados do problema podem ser utilizadas ferramentas como gráfico de Pareto, histogramas e diagrama de dispersão. Para analisar os processos utilizam-se mapas de processo e fluxogramas, por exemplo. 
Ferramentas como brainstorming, diagrama de causa e efeito, cartas de controle, testes de hipóteses, planejamento de experimentos e análise de variância são importantes para se definir as causas que mais influenciam no problema (ECKES, 2003; WERKEMA, 2004).

A quarta etapa do DMAIC é o Improve. Werkema (2004) considera que é neste momento que devem ser geradas soluções potenciais para a eliminação das causas fundamentais dos problemas detectadas na etapa anterior, bem como estas soluções devem ser testadas a fim de verificar se a solução pode ser implementada em larga escala. A autora também cita como ferramentas a serem utilizadas o brainstorming, o diagrama de causa e efeito, FMEA, simulação e 5W2H.

A etapa final do DMAIC é o Control. Para Eckes (2003) esta etapa nada mais é do que a aplicação das soluções da quarta etapa em larga escala e o controle de desempenho do processo ao longo do tempo. Werkema (2004) adiciona a padronização das alterações realizadas no processo com a adoção das soluções e a definição de um plano de ações corretivas caso surjam problemas no processo, bem como a transferência dos conhecimentos adquiridos durante o projeto para outros membros da organização.

Existem outras metodologias para implementação do Seis Sigma. PerezWilson (1999) apresenta a metodologia M/PCpS (Machine / Proces Characterization Study) e a define como uma lista de passos a serem seguidas para caracterizar e otimizar processos técnicos e não-técnicos. A M/PCps é dividia em cinco estágios, sendo que são utilizados estágios com denominações distintas para processos de manufatura e produção (técnicos) e para processos administrativos, serviços e transacionais (não-técnicos) (PEREZ-WILSON, 1999).

A metodologia DMAIC e a M/PCpS visam à melhoria da qualidade dos processos existentes. Para lidar com a qualidade de projetos de novos produtos ou serviços surgiu a metodologia Design for Six Sigma (DFSS). A aplicação desta metodologia nos projetos, preocupando-se com a qualidade desde suas primeiras fases, e garante que o processo atingirá o nível Seis Sigma quando estiver em funcionando. Conforme Prata et al. (2004), algumas vantagens desta metodologia são maior eficiência e menor custo para atingir a meta de Seis Sigma nos processos.

Independente do método adotado para implementar o Seis Sigma, o sucesso do programa depende da existência de pessoas com perfil adequado e que serão transformados em patrocinadores ou especialistas no método e nas ferramentas do 
Seis Sigma (WERKEMA, 2004). Os elementos do Seis Sigma são responsáveis pela criação, coordenação e execução dos projetos, que podem trazer benefícios para a organização, mas, em alguns casos, também podem apresentar problemas. A nomenclatura dos elementos do Seis Sigma utilizada para hierarquizar os profissionais envolvidos foi desenvolvida pelo Instituto de Pesquisa Seis Sigma da Motorola em 1991, conforme Perez-Wilson (1999).

Os elementos do Seis Sigma são denominados como Sponsor, Champion, Master Black Belt, Black Belt, Green Belt e White Belt (WERKEMA, 2004). Alguns autores, como Perez-Wilson (1999), Eckes (2001) e Gygi et al. (2005), não apresentam exatamente a mesma nomenclatura dos profissionais, mas a idéia principal e as características de cada um são similares. Uma descrição de cada um destes elementos, seguindo as diretrizes de Werkema (2004), Eckes (2001), Gygi et al. (2005) e Pande et al. (2001), é apresentada a seguir.

- Sponsor: é o executivo principal da empresa, responsável por promover e definir as diretrizes, alinhadas com a estratégia da empresa, para implementação do Seis Sigma.

- Champions: são os gestores responsáveis pela disseminação e aplicação bem sucedida do Seis Sigma, além da provisão de recursos para os projetos e da remoção das possíveis barreiras para o andamento dos projetos.

- Master Black Belts: são os profissionais coordenadores do Programa Seis Sigma que assessoram os Sponsors e Champions e prestam assistência aos Black Belts e Green Belts.

- Black Belts: são os profissionais que lideram equipes na condução de projetos e que devem possuir habilidades técnicas (métodos e ferramentas) e gerenciais.

- Green Belts: são os profissionais que participam da equipe dos Black Belts ou lideram equipes na condução de projetos funcionais.

- Yellow Belts e White Belts: são os profissionais do nível operacional da empresa, treinados nos fundamentos do Seis Sigma.

\section{METODOLOGIA}

Iniciou-se o trabalho com uma pesquisa bibliográfica sobre o Seis Sigma, com o objetivo de conhecer as contribuições existentes sobre o programa e servir de 
base para a realização do trabalho. O passo seguinte foi o planejamento, definindo o objetivo, a lista das empresas que potencialmente poderiam participar da pesquisa e o questionário. A lista das empresas a serem contatadas foi elaborada com informações de especialistas da Universidade Federal do Rio Grande do Sul, busca na Internet em sites especializados na área da Qualidade e nos sites de algumas empresas que se julgava que poderiam ter o programa implantado. O questionário foi elaborado seguindo orientações dos trabalhos de Malhotra (2001) e Gil (2007).

Visando conhecer as características gerais do programa Seis Sigma nas empresas participantes, a pesquisa teve um caráter descritivo, não sendo objetivo desta pesquisa a análise individual de cada empresa.

A pesquisa foi realizada com pessoas envolvidas com o programa Seis Sigma nas empresas que aceitaram fazer parte da pesquisa através de entrevistas pessoais ou e-mails. Para análise dos resultados obtidos foram utilizadas ferramentas estatísticas, como gráficos de Pareto, por exemplo, e análises qualitativas das questões abertas, utilizando as recomendações da análise de conteúdo proposta por Bardin (1977).

\section{DESENVOLVIMENTO DO ESTUDO}

O trabalho foi desenvolvido seguindo as etapas mostradas na Figura 1. Após a revisão bibliográfica foi elaborado um questionário para levantamento das características que se desejava conhecer do programa Seis Sigma. Em seguida, foram contatadas algumas empresas para convidá-las a participar da pesquisa. A etapa seguinte é a análise dos dados e apresentação dos resultados para que, na última etapa, seja possível realizar a conclusão do trabalho. A descrição destas etapas é realizada na seqüência deste artigo.

Figura 1 - Etapas de desenvolvimento do estudo

1. Elaboração do
Questionário $\rightarrow \begin{gathered}\text { 2. Contato com } \\ \text { as empresas }\end{gathered} \rightarrow \begin{gathered}\text { 3. Aplicaçấo do } \\ \text { questionário }\end{gathered} \rightarrow \begin{gathered}4 \text {. Resultados } \\ \text { da pesquisa }\end{gathered}$

\subsection{Elaboração do questionário}

O questionário de diagnóstico foi desenvolvido visando traçar um perfil do 
programa Seis Sigma de empresas da região metropolitana de Porto Alegre. Ele é composto por três blocos denominados, respectivamente, dados da empresa, Seis Sigma e especialistas do Seis Sigma. O cabeçalho não foi incluído no questionário, embora seja o usual, devido ao fato dele ter sido aplicado em 3 empresas através de entrevista pessoal, onde a pesquisadora esclareceu os objetivos da pesquisa antes do início da entrevista, ou via Internet, onde foi enviado um e-mail explicativo. A Figura 2 apresenta este questionário na íntegra.

No primeiro bloco do diagnóstico foram solicitadas informações gerais da empresa, como segmento de atuação e a nacionalidade da empresa, por exemplo. Com estes dados é possível avaliar a origem e o porte das empresas, bem como os setores econômicos onde o programa é mais utilizado.

O segundo bloco solicita informações sobre o programa Seis Sigma. Levantando questão referente ao ano de implantação do programa; ao método de aplicação do programa na empresa; às ferramentas estatísticas e gerenciais mais utilizadas; às certificações de programas de qualidade que a empresa possui; às razões que levaram a empresa a implantar o programa e as áreas da empresa onde o programa está em funcionamento.

O terceiro bloco deste diagnóstico visava obter informações sobre os especialistas do Seis Sigma nas empresas, os Belts. O primeiro item questionado foi a quantidade de Master Black Belt, Black Belt e Green Belts que a empresa possui em atividade. Em seguida, procurou-se conhecer a formação acadêmica destes especialistas e quais os critérios utilizados pela empresa para selecioná-los. O tempo de dedicação e a quantidade de horas de treinamento também foram informações solicitadas neste bloco.

O pré-teste deste questionário foi realizado com o especialista da primeira empresa a participar da pesquisa (Empresa $n^{\circ} 3$ ), que possui certificação da $A S Q$ (American Society for Quality) como Master Black Belt e que coordena o programa da empresa na América Latina, possuindo grande experiência no programa Seis Sigma. As considerações sobre o questionário durante esta entrevista foram importantes, mas não alteraram significativamente o conteúdo do questionário, sendo que este foi aplicado posteriormente nas demais empresas. 
Figura 2 - Questionário utilizado na pesquisa

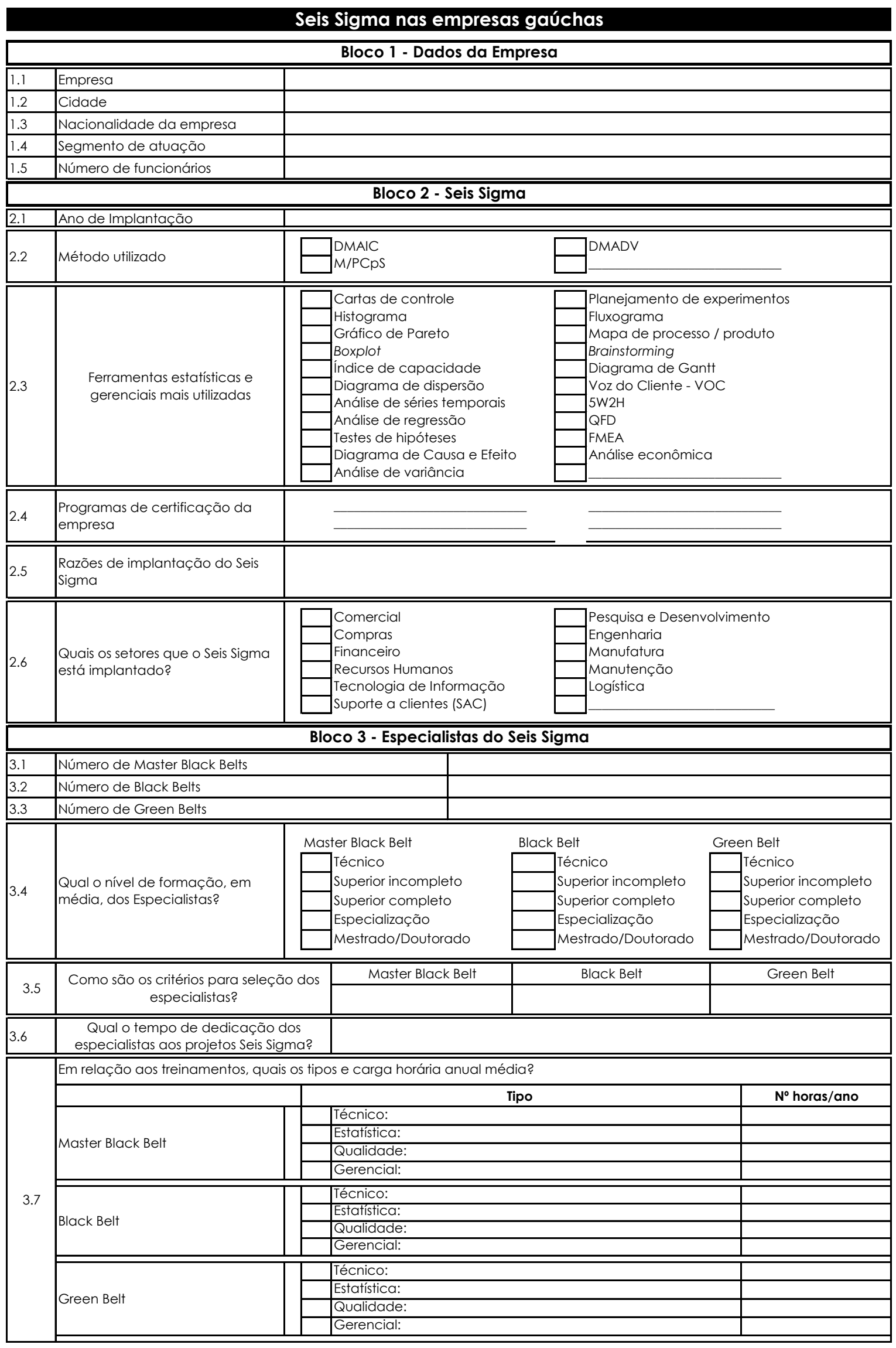




\subsection{Contato com as empresas}

Os critérios para seleção destas empresas foram: (i) sua localização na região metropolitana de Porto Alegre e (ii) a existência do programa Seis Sigma. Inicialmente buscou-se informação junto aos especialistas da área na Universidade Federal do Rio Grande do Sul (UFRGS) para descobrir algumas empresas da região geográfica de interesse que utilizavam o programa Seis Sigma e onde se conhecia alguém para realizar o primeiro contato, chamado aqui de facilitador.

O contato inicial com as empresas foi efetivado via e-mail nos casos onde um facilitador poderia fornecer o endereço eletrônico de uma pessoa de contato dentro da empresa candidata. Nos demais casos, o contato inicial foi realizado através de uma ligação telefônica para a empresa, sendo solicitado o atendimento pelo responsável pelo Seis Sigma ou pela área da Qualidade da organização. Após aceitação da empresa, o questionário foi enviado juntamente com um e-mail explicativo contendo o objetivo da pesquisa. Cabe ressaltar que, em três destas empresas este foi aplicado através de entrevistas pessoais.

As entrevistas pessoais iniciaram-se com a apresentação da empresa e do programa Seis Sigma por um especialista da área e em seguida foram solicitadas as informações do questionáro, sendo que estas entrevistas não foram gravadas. Participaram efetivamente da pesquisa onze empresas, sendo estas numeradas de 1 a 11, como forma de identificação nesta pesquisa.

\subsection{Aplicação dos questionários}

A realização do diagnóstico do programa Seis Sigma nas empresas participantes da pesquisa iniciou-se com a realização de entrevistas com representantes do programa em 3 empresas identificadas como empresas $n^{0} 3,6$ e 9. Nas demais empresas os questionários foram enviados por e-mail e, posteriormente, devolvidos devidamente preenchidos, também via e-mail. Visto que as entrevistas com os especialistas das empresas contribuíram para a construção do trabalho, estas serão descritas nesta seção.

A primeira entrevista foi realizada na empresa $n^{\circ} 3$ e teve duração aproximada de 2 h30min. A visita foi realizada em Julho de 2008 e a pessoa que participou da entrevista ocupa a posição de Master Black Belt (MBB). Durante esta visita foi 
realizado o pré-teste dos questionários, o que contribuiu significativamente para o resultado da pesquisa.

Foi relatado pelo MBB que o programa Seis Sigma da empresa teve início, no Brasil, na fábrica de Gravataí, em 2000. A necessidade de melhoria da qualidade dos produtos e a redução do desperdício, além da busca da sobrevivência da empresa, foram os motivos que levaram a organização a implantar o Seis Sigma. A estrutura do Seis Sigma desta organização no Brasil é composta por três MBB, sendo que um deles tem ligação direta com a Vice-presidência da empresa. O canal direto com a Vice-presidência é fundamental para que os projetos sejam alinhados com as estratégias da empresa e que tenham o apoio da diretoria.

$\mathrm{Na}$ época da entrevista, a empresa estava planejando a implantação do Seis Sigma na área administrativa, pois até o momento somente a produção (manufatura e engenharia) de algumas unidades tem o programa fortemente disseminado. As primeiras iniciativas do Seis Sigma tiveram muitos obstáculos, uma vez que a direção ainda não estava convicta dos benefícios que o programa poderia trazer. Somente após a matriz evidenciar a necessidade de implantação do Seis Sigma é que realmente o programa foi efetivado e conseguiu obter sucesso, tendo alcançado resultados financeiros acima do planejado em alguns projetos. Outra característica citada durante a entrevista foi a rotatividade dos especialistas nos primeiros anos do Seis Sigma na empresa, uma vez que o mercado não possuía profissionais habilitados e outras empresas da região iniciaram o processo de implantação do programa de qualidade, atraindo alguns profissionais já treinados pela empresa 3.

A segunda visita foi à empresa $n^{\circ} 6$ em Agosto de 2008, com duração aproximada de $1 \mathrm{~h} 30 \mathrm{~min}$. A entrevista foi realizada com o Master Black Belt da unidade de Canoas. O programa iniciou em 2000 na empresa e hoje está implantado em todos os seus setores, o que é um diferencial em comparação com as demais empresas participantes da pesquisa. A empresa desenvolveu um sistema de treinamento on-line, via intranet, sobre conceitos e ferramentas básicas do Seis Sigma. O objetivo deste treinamento é disseminar a cultura do Seis Sigma em toda empresa e tem duração de 5h/aula, já tendo formado aproximadamente 1500 funcionários como White Belt. Para os novos funcionários, este treinamento é obrigatório, para os demais é opcional.

Todo o sistema de apresentação, aprovação e acompanhamento dos projetos do Seis Sigma da empresa $n^{\circ} 6$ é realizado via intranet. O MBB é responsável pela 
aprovação e acompanhamento dos resultados dos projetos, bem como pelo auxílio para obter a solução de problemas que possam surgir durante a implementação dos projetos. Outro item bastante valorizado, e que inclusive consta na Visão de Futuro da empresa, é o reconhecimento do desempenho dos colaboradores. Para isso, a organização possui programas motivacionais, onde as competências-chave dos colaboradores são reconhecidas e premiadas com benefícios financeiros.

A terceira e última visita foi com a Black Belt (BB) da unidade de Gravataí da empresa $n^{\circ}$ 9. Com duração aproximada de $2 \mathrm{~h}$, a entrevista realizada em Agosto de 2008 foi muito rica, pois esta empresa implantou o Seis Sigma em 2006 e o programa ainda não está consolidado como nas outras duas empresas visitadas. A estrutura do Seis Sigma ainda não conta com MBB e atualmente a função de coordenação do programa encontra-se a cargo da BB que participou da entrevista.

As maiores dificuldades citadas pela BB durante a entrevista referiram-se aos recursos humanos, geralmente escassos e, às vezes, com qualidade abaixo da que a empresa deseja. Outro fator ressaltado foi a falta de um plano de carreira para os Belts, oferecendo perspectivas de crescimento profissional para estes especialistas. Um problema citado durante a entrevista foi a disponibilidade de tempo dos Belts ser parcial para condução dos projetos do Seis Sigma, o que pode ocasionar atrasos e resultados não satisfatórios com os projetos.

A empresa $n^{\circ} 9$ utiliza consultorias externas para consultas técnicas a respeito da metodologia e ferramentas estatísticas complexas, diferenciando-se das empresas que possuem o Seis Sigma implantado há mais tempo e que possuem um corpo de especialistas maior e mais experiente para atender as necessidades da empresa.

\subsection{Resultados da pesquisa}

O questionário para diagnóstico, apresentado na figura 2, apresenta três blocos. Os resultados obtidos são apresentados para cada um dos blocos, conforme divisão proposta no questionário.

\subsubsection{Bloco 1 - Dados da empresa}

Do primeiro bloco de questões, foram obtidas as seguintes informações: 
a) Das 11 empresas participantes, 5 são subsidiárias de empresas com matriz nos Estados Unidos, o que representa $45 \%$ das empresas pesquisadas.

b) A cidade com o maior número de empresas participantes é Gravataí, onde quatro organizações contribuíram com a pesquisa.

c) As empresas que se encontram dentro dos setores automotivo e agrícola, ou em setores ligados a eles como: pneumáticos; motores e componentes eletrônicos foram as mais freqüentemente encontradas nesta pesquisa.

d) Considerando a classificação do porte das empresas pelo número de empregados, percebe-se que apenas uma das empresas pesquisadas enquadrase na categoria de média empresa (até 500 funcionários). As demais são todas grandes empresas.

\subsubsection{Bloco 2 - Seis Sigma}

O bloco seguinte refere-se ao programa Seis Sigma e suas características nas empresas. A primeira constatação ao observar os dados é que $91 \%$ das empresas pesquisadas utilizam como método o DMAIC (Define, Measure, Analyze, Improve e Control). Este resultado é semelhante ao obtido no trabalho realizado por Andrietta e Miguel (2007), onde aproximadamente 95\% das empresas participantes utilizam o DMAIC.

Para 3 das empresas pesquisadas o programa Seis Sigma teve início em 2000 , o que representou $27 \%$ das empresas participantes. Na maior parte das empresas, 64\%, o programa teve início a partir de 2003, evidenciando que o Seis Sigma é um programa novo e em estruturação nestas empresas, não estando completamente consolidado em algumas delas.

Em relação às ferramentas utilizadas pelas empresas, algumas são amplamente utilizadas, como: FMEA, Mapas de Processo, Diagrama de Causa e Efeito, Gráfico de Pareto e Cartas de Controle. Em contrapartida, ferramentas poderosas e que poderiam agregar mais valor aos produtos, principalmente por basearem-se em informações dos clientes, como Voz do Cliente e QFD, têm sua utilização restrita a poucas empresas. Outra ferramenta poderosa, a análise de séries temporais, só foi citada como instrumento utilizado por duas empresas. $\mathrm{O}$ Gráfico de Pareto que ilustra estas conclusões encontra-se na Figura 3. Uma 
explicação para o uso de algumas destas ferramentas na maior parte das empresas talvez esteja relacionada com exigências de programas de certificação estas empresas possuem, como, por exemplo, a TS16949.

Em relação aos programas de certificação das empresas participantes podese afirmar que todas possuem certificações no Sistema de Gestão da Qualidade (ISO 9001) e no Sistema de Gestão Ambiental (ISO 14001). Além disso, todas as empresas que atuam no setor automobilístico, ou fornecendo produtos para ele, o que representa $55 \%$ das empresas pesquisadas, possuem a certificação da norma automotiva mundial TS 16949. Já a certificação do Sistema de Gestão da Segurança e Saúde Ocupacional (OHSAS 18001) foi apresentada por 6 empresas (55\%), porém não se restringindo às empresas do setor automobilístico.

Figura 3 - Ferramentas utilizadas pelas empresas participantes

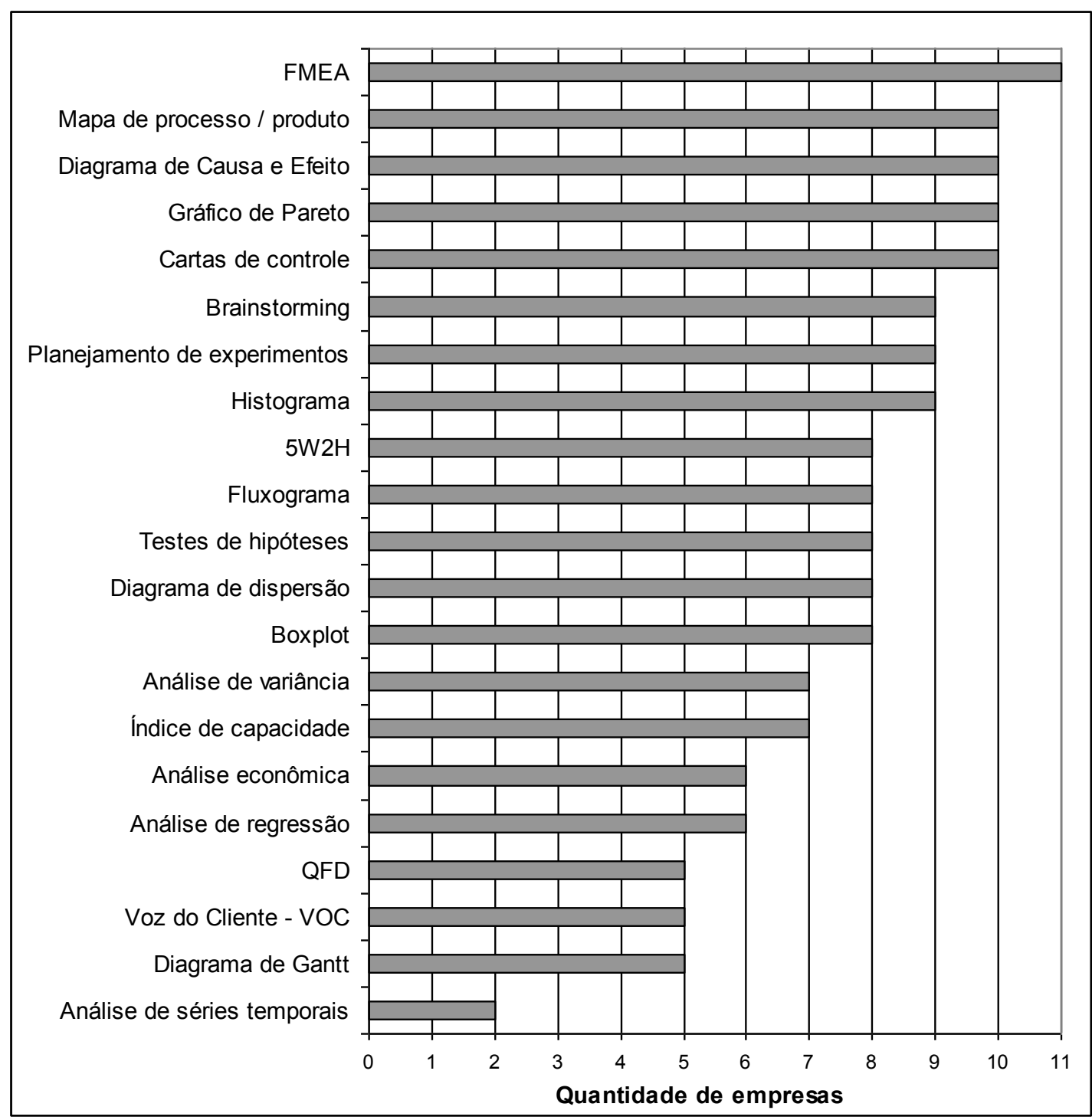


Através de uma análise similar à análise de conteúdo proposta por Bardin (1977) foi possível verificar quais as principais razões que levaram as empresas a implantar o programa Seis Sigma. Do resultado mostrado na Figura 4 percebe-se que a melhoria dos processos, a melhoria da qualidade dos produtos e a redução dos desperdícios foram os motivos que determinaram a implantação do Seis Sigma em mais empresas. Somente uma empresa apontou como causa de implantação a determinação por parte da matriz, o que difere do resultado da pesquisa realizada por Andrietta e Miguel (2007), onde 50\% das empresas pesquisadas na ocasião apontaram a decisão da matriz como causa determinante para implantação do Seis Sigma.

Figura 4 - Principais causas de implantação do Seis Sigma

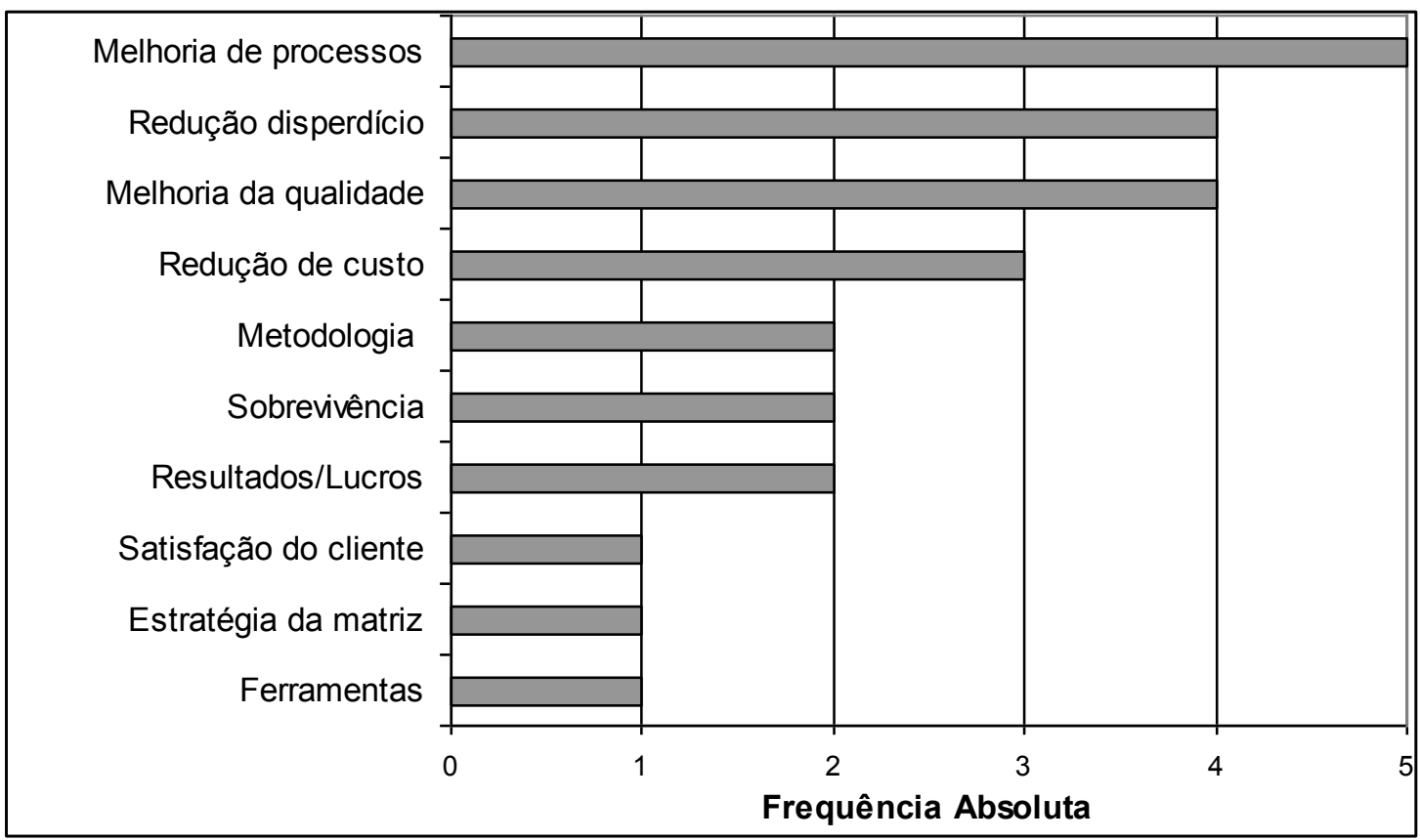

Em relação aos setores, percebe-se que a Manufatura é o único setor onde todas as empresas participantes utilizam o Seis Sigma. A Empresa 6 é a única onde o Seis Sigma está implantado em todos os setores, nas demais o programa está sendo utilizado parcialmente. É possível notar também que nas áreas correlacionadas com a Manufatura, como Engenharia, Compras, Manutenção, Logística e Pesquisa e Desenvolvimento, o programa tem mais penetração. É evidenciado também que setores administrativos, como Financeiro, Comercial e Recursos Humanos, ainda não estão utilizando o programa, mostrando que existe ainda um vasto campo de aplicação a ser explorado. 


\subsubsection{Bloco 3 - Especialistas do Seis Sigma}

O terceiro bloco do questionário para diagnóstico do Seis Sigma nas empresas estudadas trata dos Especialistas. Das 11 empresas pesquisadas, apenas 4 (36\%) possuem Master Black Belt (MBB). Nas empresas onde não existe a figura do MBB, a função de coordenação e de apoio com as ferramentas gerenciais e estatísticas é realizada por Black Belt ou por consultoria externa. Em apenas uma empresa não existe nem mesmo Black Belt, sendo que todo o andamento do programa está sob responsabilidade de Green Belts.

A relação entre o número de Black Belts (BB) e Green Belts (GB) apresentou grande variação. Em duas empresas existem mais Black Belts que Green Belts, invertendo a recomendação da literatura. Na empresa $\mathrm{n}^{\circ} 2$ só existem Green Belts, portanto não foi possível obter a proporção GB/BB. Nas demais empresas (73\%) foi verificado que a proporção GB/BB é maior que um, mas não foi possível verificar uma constância nos valores. A Figura 5 ilustra a relação GB/BB para cada empresa sendo que a média destes valores é 6,38.

Figura 5 - Relação entre o número de Green Belts e Black Belts nas empresas pesquisadas

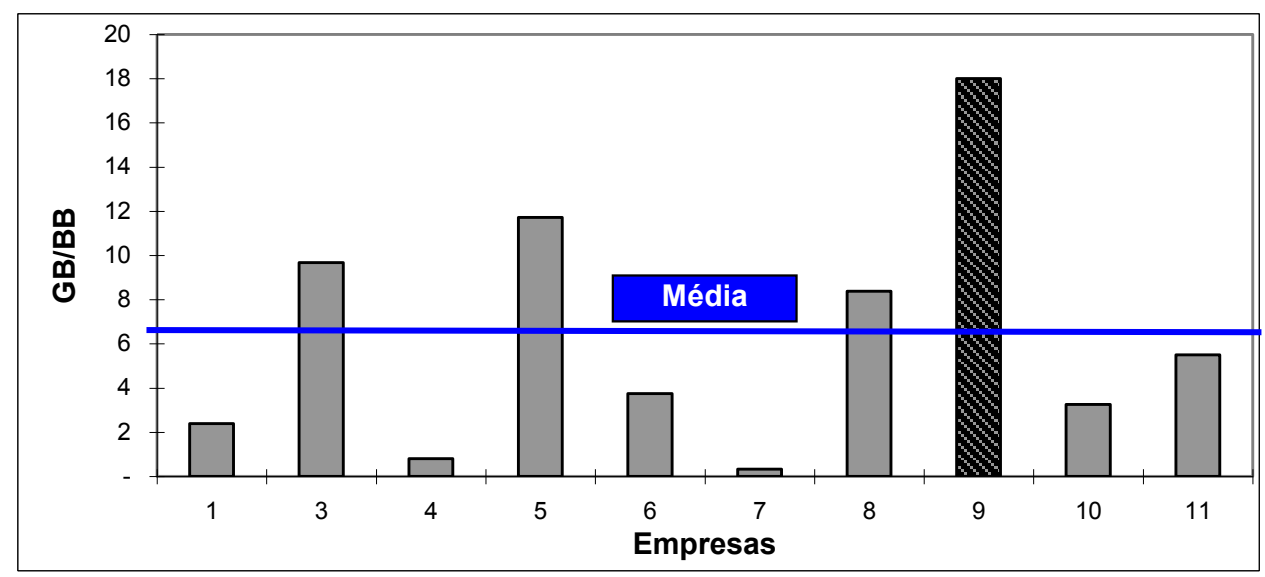

Voltando a atenção para a formação do corpo de especialistas das empresas participantes da pesquisa é possível verificar o seguinte: (i) os MBB possuem especialização ou mestrado; (ii) os $\mathrm{BB}$, em sua maioria (79\%), possuem curso superior completo ou especialização; (iii) os GB, em sua maioria (73\%), possuem curso superior completo ou incompleto. É possível notar algumas discrepâncias em relação à formação dos especialistas. Por exemplo, em uma das empresas existe Black Belt com formação de nível técnico e em outra os Green Belts possuem até 
mesmo curso de especialização. Uma constatação é que não existe relação entre certificação e formação acadêmica que possa ser generalizada para todas as empresas participantes.

Nas empresas pesquisadas não foi possível evidenciar critérios claros e uniformes para a construção do corpo de especialistas do Seis Sigma, resultado que reforça o apresentado por Kessler (2004), que verificou que não há critérios semelhantes para seleção dos gerentes de projeto nas empresas que participaram de sua pesquisa. Para os Green Belts, o processo de seleção ainda está alicerçado na indicação gerencial e no perfil do candidato. Também foram citados como critérios o bom aproveitamento do curso de formação, a colaboração em projetos executados e o conhecimento da metodologia e das ferramentas estatísticas. Uma das empresas utiliza como critério o colaborador dispor de tempo para participar do programa e outra define os especialistas conforme o tipo de projetos que será implantado.

Para os Black Belts já existem critérios claramente definidos em algumas empresas. Na empresa 3, por exemplo, o candidato a BB precisa ser Green Belt, ter bom inglês técnico e conhecimento da metodologia do Seis Sigma, além da aprovação em uma prova teórica, em uma dinâmica de grupo e em uma entrevista com seus superiores hierárquicos do Seis Sigma. São características dos BBs, citadas pelas empresas com maior freqüência, o bom desempenho como GB, capacidade de liderança, bom conhecimento da metodologia, das ferramentas estatísticas e dos processos internos da empresa. Critérios como indicação gerencial e adequação aos projetos que serão desenvolvidos pela empresa ainda apareceram para definição das pessoas que terão esta função de especialista. Já para os Master Black Belts apareceram critérios mais técnicos e precisos como, por exemplo, capacitação técnica, habilidade de liderança de equipes e obtenção de certificação de órgão externo.

Nas empresas que possuem MBB, estes especialistas atuam em tempo integral no programa Seis Sigma, diferindo da pesquisa de Andrietta e Miguel (2007), onde esse percentual era de apenas 35\%. Em quatro empresas, os BB também atuam durante todo o tempo nos projetos Seis Sigma, porém nas demais empresas apenas parte do tempo é dedicada ao programa, algo em torno de $50 \%$ do tempo destes especialistas. Já para os GB o tempo é parcial em todas as empresas que responderam a esta questão, variando entre $20 \%$ e $30 \%$ do tempo na maior parte 
dos casos.

Considerando a questão dos Treinamentos, verificou-se que em 4 empresas o treinamento básico sobre o Seis Sigma é oferecido a todos os funcionários. $\mathrm{Na}$ empresa 6 o treinamento de White Belt (Básico) é ofertado via intranet a todos interessados, porém é obrigatório para os novos funcionários e opcional para os antigos. O fato que ficou evidenciado pela pesquisa é que a maioria das empresas oferece apenas o curso de formação para os Belts, não havendo preocupação em reciclagem e treinamento contínuo. Somente uma empresa oferta 8h/ano de treinamento para os Green Belts sobre ferramentas e técnicas estatísticas, como forma de atualização e aprendizado contínuo. A média de horas dos cursos de formação dos especialistas é de 48hs para os Green Belt, de $120 \mathrm{~h}$ para os Black Belts e de 210h para os Master Black Belts.

\section{CONCLUSÕES}

Durante a realização do contato inicial com as empresas, salienta-se a receptividade da maioria das pessoas, que se mostraram interessadas na pesquisa e se dispuseram a fornecer informações e a responder aos questionários. Além disso, estas pessoas se empenharam em atender ao cronograma da pesquisa, dedicando parte de seu tempo para compartilhar suas experiências e conhecimentos, pois sem isso, com certeza, esse trabalho não teria obtido os resultados aqui apresentados.

O primeiro resultado obtido com a aplicação do procedimento proposto foi o mapeamento do Seis Sigma nas empresas participantes, evidenciando características do programa nestas empresas. Nesta etapa foi evidenciado que as empresas que participaram da pesquisa implantaram o programa Seis Sigma para obter melhorias em seus processos produtivos, reduzir desperdícios e melhorar a qualidade de seus produtos. Este resultado expressa a preocupação das empresas pesquisadas em: obter melhores resultados financeiros e, também atender as necessidades de seus clientes.

Percebe-se nos dados obtidos com o diagnóstico do Seis Sigma que o método DMAIC é utilizado pela maioria das empresas pesquisadas e que todas estas empresas possuem certificações ISO 9001 e 14001. Observando os resultados nota-se que o Seis Sigma não está difundido nas áreas administrativas 
da maioria das empresas pesquisadas e que ainda está restrito á áreas como manufatura e engenharia. O quadro 1 apresenta uma comparação entre os resultados da pesquisa e o constante na literatura.

Quadro 1 - Comparação entre dados da literatura e resultados da pesquisa

\begin{tabular}{|c|c|c|}
\hline $\begin{array}{c}\text { Fator de } \\
\text { comparação }\end{array}$ & Literatura & Resultado da Pesquisa \\
\hline $\begin{array}{l}\text { Características } \\
\text { das empresas } \\
\text { pesquisadas }\end{array}$ & $\begin{array}{l}\text { - Andrietta e Miguel (2007): } 65 \% \text { das empresas } \\
\text { pesquisadas são de grande porte; } 37 \% \text { são do } \\
\text { setor automotivo. }\end{array}$ & $\begin{array}{l}\text { - 91\% das empresas são de grande porte; Setor } \\
\text { automotivo e agrícola foram os mais frequentes } \\
\text { na pesquisa }\end{array}$ \\
\hline Método DMAIC & $\begin{array}{l}\text { - Andrietta e Miguel (2007): 95\% das empresas } \\
\text { pesquisadas utilizavam o DMAIC. } \\
\text { - Werkema (2004) e Eckes (2003): método mais } \\
\text { utilizado é o DMAIC. }\end{array}$ & $\begin{array}{l}\text { - } 91 \% \text { das empresas pesquisadas utilizavam o } \\
\text { DMAIC }\end{array}$ \\
\hline $\begin{array}{l}\text { Motivo para } \\
\text { implantação do } \\
\text { Seis Sigma }\end{array}$ & $\begin{array}{l}\text { - Andrietta e Miguel (2007): } 50 \% \text { das empresas } \\
\text { pesquisadas implantaram o Seis Sigma por } \\
\text { decisão da matriz }\end{array}$ & $\begin{array}{l}\text { - Somente } 9 \% \text { das empresas implantaram o } \\
\text { Seis Sigma por decisão da matriz; } 45 \% \\
\text { implantaram para melhoria dos processos }\end{array}$ \\
\hline $\begin{array}{l}\text { Especialistas do } \\
\text { Seis Sigma }\end{array}$ & $\begin{array}{l}\text { - Kessler (2004): Não há critérios para seleção } \\
\text { de gerentes de projetos } \\
\text { - Santos e Martins (2010): Existência de critérios } \\
\text { definidos para selação de BB nas duas } \\
\text { empresas pesquisadas. } \\
\text { - Andrietta e Miguel (2007): 35\% dos MBB atuam } \\
\text { em tempo integral no Seis Sigma. } 40 \% \text { das } \\
\text { empresas tem MBB. } \\
\text { - Gonçalves e Musetti (2008): Não há dedicação } \\
\text { exlcusiva ao Seis Sigma nas empresas } \\
\text { pesquisadas. } 90 \% \text { dos colaboradores são } \\
\text { treinados no Seis Sigma em uma das empresas } \\
\text { pesquisadas. }\end{array}$ & $\begin{array}{l}\text { - Não há critérios claros e definidos para } \\
\text { construção do quadro de especialistas. } \\
\text { - Nas empresas que possuem MBB, estes } \\
\text { atuam em tempo integral. Os BB atuam em } \\
\text { aproximadamente } 50 \% \text { do seu tempo no Seis } \\
\text { Sigma. } \\
\text { - } 36 \% \text { das empresas pesquisadas tem MBB. } \\
\text { - Treinamento básico do Seis Sigma para todos } \\
\text { os funcionários em } 36 \% \text { das empresas } \\
\text { pesquisadas. } \\
\text { - Maioria das empresas oferece apenas } \\
\text { treinamento de formação no programa, não } \\
\text { havendo previsão de reciclagens. }\end{array}$ \\
\hline
\end{tabular}

Outro resultado importante desta fase refere-se aos Belt, especificamente à falta de um plano de carreira com critérios definidos para avanços na hierarquia do programa, uma vez que não foi possível estabelecer um padrão de conhecimentos, habilidades e atitudes que pudesse ser adotado em todas as empresas pesquisadas para definir o perfil de cada um dos Belts. Salienta-se também a escassez de treinamentos de atualização para os especialistas do programa, nas empresas pesquisadas, após os cursos de formação.

Por se tratar de pesquisa com uma abordagem qualitativa, os resultados apresentados não podem ser generalizados, o que pode ser considerado uma limitação, porém não invalidam os resultados aqui apresentados considerando as onze empresas participantes. 


\section{REFERÊNCIAS}

ANDRIETTA, João Marcos; MIGUEL, Paulo Augusto Cauchick. Aplicação do programa Seis Sigma no Brasil: resultados de um levantamento tipo survey exploratório-descritivo e perspectivas para pesquisas futuras. Gestão \& Produção, São Carlos, v. 14, n. 2, p. 203-219, maio/ago. 2007.

ANTONY, Jiju; BANUELAS, Ricardo. Key ingredients for the effective implementation of Six Sigma program. Measuring Business Excellence, v. 6, n. 4, p. 20-27, 2002.

ANTONY, Jiju; Some pros and cons of Six Sigma: an academic perspective. The TQM Magazine, v. 16, n. 4, p. 303-306, 2004.

BARDIN, L. Análise de Conteúdo. 70. ed. São Paulo: Livraria Martins Fontes, 1977.

CARVALHO, Marly Monteiro de; HO, Linda Lee; PINTO, Silvia Helena Boarin. Implementação e difusão do programa Seis Sigma no Brasil. Prod. [online], V.17, n.3, p. 486-501, 2007.

CABRERA JR, Álvaro. Dificuldades de implementação de programa Seis Sigma: estudos de casos em empresas com diferentes níveis de maturidade.

Dissertação de Mestrado. Escola de Engenharia de São Carlos. Universidade de São Paulo. São Carlos, 2006.

ECKES, George. A Revolução do Seis Sigma: o método que levou a GE e outras empresas a transformar processos em lucro. Rio de Janeiro: Campus, 2001.

Six Sigma for Everyone. Hoboken: John Wiley \& Sons, 2003.

FEE - Fundação de Economia e Estatística. PIB Estadual - Desempenho anual. Disponível em:

http://www.fee.tche.br/sitefee/pt/content/estatisticas/pg_pib_estado_desempenho.ph p Atualizado em: Dezembro 2006. Acesso em: 11 set. 2010.

FIERGS - Federação das Indústrias do Rio Grande do Sul. Indicadores Industriais - Novembro de 2009. Disponível em: http://www.fiergs.org.br/canais_fiergs.asp ?idArea=48\&idSubMenu=65\&idSubSubMenu=271\&idSubSubSubMenu=276. Acesso em: 18 fev. 2010.

GIL, Antonio Carlos. Métodos e técnicas em pesquisa social. 5. ed. São Paulo: Atlas, 2007.

GYGI, Craig; DECARLO, Neil; WILLIAMS, Bruce. Six Sigma for Dummies. Hoboken, N.J: Wiley, 2005.

GONÇALVES, Bianca Soares de Oliveira; MUSETTI, Marcel Andreotti. A importância do processo de alinhamento da estratégia com projetos Seis Sigma: um estudo multicasos em operadores logísticos. Gest. Prod. [online], v.15, n.3, p. 551-562, 2008. 
JURAN, Joseph. M. Planejando para a qualidade. 3. ed. São Paulo: Pioneira, 1995.

KESSLER, Rafael Motta. A implantação do Seis Sigma em organizações:

motivações de escolha e resultados obtidos. Dissertação (Mestrado profissional). Universidade Federal do Rio Grande do Sul. Programa de Pós-graduação em Administração. Porto Alegre, 2004.

MALHOTRA, Naresh. Pesquisa de marketing: uma orientação aplicada. 3. ed. Porto Alegre: Bookman, 2001.

MERGULHÃO, Ricardo Coser. Análise da implementação do Seis Sigma em empresas de manufatura no Brasil. Itajubá: UNIFEI, 2003. 89 p. (Dissertação de mestrado apresentada ao Programa de Pós-Graduação em Engenharia de Produção da Universidade Federal de Itajubá).

OLIVEIRA, Marcelle Colares; DAHER, Wilton de Medeiros. A utilização da metodologia seis sigma em empresa do setor hoteleiro um estudo de caso.

ENCONTRO NACIONAL DE ENG. DE PRODUÇÃO, 24. 2004. Anais. Florianópolis, 2004.

PANDE, Peter S.; NEUMAN, Robert P.; CAVANAGH, Roland R. Estratégia Seis Sigma: como a GE, a Motorola e outras grandes empresas estão aguçando seus desempenho. Rio de Janeiro: Qualitymark Ed., 2001.

PEREZ-WILSON, Mario. Seis Sigma: compreendendo o conceito, as implicações e os desafios. Rio de Janeiro: Qualitymark Ed.,1999.

PRATA, Rodrigo Francisco; FERNANDES, Tatiana; SILVA, Carlos Eduardo Sanches da; TURRIONI, João Batista. Fundamentos do design for six sigma. SIMPEP, 11. 2004. Anais. Bauru, SP, 2004.

SANTOS, Adriana Barbosa; MARTINS, Manoel Fernando. Contribuições do Seis Sigma: estudo de caso em multinacionais. Produção, v.20, n.1, jan./mar. 2010.

SLACK, Nigel; CHAMBERS, Stuart; HARLAND, Christine; HARRISON, Alan; JOHNSTON, Robert. Administração da Produção. São Paulo: Atlas, 2006.

WERKEMA, Maria Cristina Catarino. Criando a cultura Seis Sigma. Nova Lima, MG: Werkema Ed., 2004.

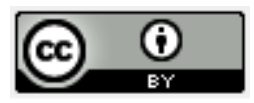

Artigo recebido em 25/03/2010 e aceito para publicação em 23/01/2012. 